\title{
Alpha-decay energies of superheavy nuclei for the Fayans functional
}

\section{Tolokonnikov, S. V.}

2017-02-23

Tolokonnikov , S V , Borzov , I N , Kortelainen , M , Lutostansky, Y S \& Saperstein , E E 2017 , ' Alpha-decay energies of superheavy nuclei for the Fayans functional ' , European Physical Journal. A, Hadrons and Nuclei , vol. 53 , no. 2 , 33 . https://doi.org/10.1140/epja/i2017-12220-y

http://hdl.handle.net/10138/312417

https://doi.org/10.1140/epja/i2017-12220-y

acceptedVersion

Downloaded from Helda, University of Helsinki institutional repository.

This is an electronic reprint of the original article.

This reprint may differ from the original in pagination and typographic detail.

Please cite the original version. 


\title{
Alpha-decay energies of superheavy nuclei for the Fayans functional
}

\author{
S. V. Tolokonnikov \\ National Research Centre "Kurchatov Institute", 123182 Moscow, Russia and \\ Moscow Institute of Physics and Technology, Dolgoprudny \\ I. N. Borzov \\ National Research Centre "Kurchatov Institute", 123182 Moscow, Russia and \\ Joint Institute for Nuclear Research, 141980 Dubna, Russia \\ M. Kortelainen \\ Department of Physics, P.O. Box 35 (YFL), University of Jyvaskyla, FI-40014 Jyvaskyla, Finland and \\ Helsinki Institute of Physics, P.O. Box 64, FI-00014 University of Helsinki, Finland \\ Yu. S. Lutostansky \\ National Research Centre "Kurchatov Institute", 123182 Moscow, Russia \\ E. E. Saperstein \\ National Research Centre "Kurchatov Institute", 123182 Moscow, Russia and \\ National Research Nuclear University MEPhI, 115409 Moscow, Russia
}

\begin{abstract}
Alpha-decay energies for several chains of super-heavy nuclei are calculated within the selfconsistent mean-field approach by using the Fayans functional FaNDF ${ }^{0}$. They are compared to the experimental data and predictions of two Skyrme functionals, SLy4 and SkM*, and of the macro-micro method as well. The corresponding lifetimes are calculated with the use of the semiphenomenological formulas by Parkhomenko and Sobiczewski and by Royer and Zhang.
\end{abstract}

PACS numbers: 21.60.Jz, 21.10.Ky, 21.10.Ft, 21.10.Re

\section{INTRODUCTION}

During the last decade, remarkable progress has been achieved in a synthesis of superheavy nuclei belonging to the so-called "stability island", the elements with charge numbers $Z \simeq 114-120$, predicted about fifty years ago [1, 2]. Two isotopes of the element with $Z=116$, named later Lv, and one with $Z=118$ were created first by Oganessian et. al. [3]. After the element 118 two isotopes of the element $Z=117$ were synthesized [4]. The latter element was investigated in more details in Refs. [5 8] An attempt [9] to create the element with $Z=120$ turned out to be unsuccessful, however, evidently, efforts in this direction will be continued.

In fact, the stability island turned out to be "the stability shallow", as all of these new nuclei are not stable. Although their lifetimes are large in the "nuclear scale", they are usually not sufficiently long lived to be detected in a usual way. All of them undergo alpha-decay, and the authors of the experimental works cited above detected, for each primary nucleus, a chain of several alpha-decays by measuring the alpha-decay energies $E_{\alpha}$ with high accuracy, and the respective lifetimes $T_{\alpha}$, leading to a nucleus which was already known. Therefore, a theoretical support is desirable to make such kind of indirect identification of the new superheavy nuclei more reliable.

For even-even nuclei, in which the transition occurs between the $0^{+}$ground states, the alpha-decay energies $E_{\alpha}(Z, N)$ are determined, with allowance for the recoil effect, in terms of the mass difference between nuclei related by alpha decay:

$$
Q_{\alpha}(Z, N)=M\left({ }_{Z}^{A} X_{N}\right)-M\left({ }_{Z-2}^{A-4} Y_{N-2}\right)-M_{\alpha} .
$$

For odd and odd-odd nuclei, a correction to this simple formula could appear due to a possible excitation of the parent and/or daughter nucleus which may occur in the real experimental situation.

In Refs. [3 7], the experimental data for $Q_{\alpha}$ were compared with predictions [10, 11] of the so-called macromicro method (MMM) 12, 13]. In this method, the binding energy of a nucleus is found as the sum of two terms, a macroscopic energy which was calculated on the basis of the liquid-drop model and a shell correction energy which was found according to the Strutinsky method [2]. Thus, two independent sets of parameters are used in the MMM, one for the nuclear droplet model and the second for the Shell Model potential. In general, such a comparison confirmed the identification of new superheavy nuclei. Recently, in a comprehensive study of superheavy nuclei 14], predictions of the MMM, including $\alpha$-decay characteristics, were compared to those from the nuclear energy density functional (EDF) method. Two Skyrme EDFs were used, SkM* [15] and SLy4 [16], for which the parametrizations can be also found in the review article 17]. A relativistic EDF DD-PC1 18] was also used for a comparison. The main bulk of calculations was carried out within the self-consistent mean-field (SCMF) approach. In addition, several examples of beyond mean-field results were presented which explicitly 
considered collective correlations related to the symmetry restoration and fluctuations of the collective coordinates. It was shown that the SCMF described well $\alpha$-decay and $\beta$-decay energies. At the same time, beyond mean-field corrections are important in order to find the correct deformation energy curves and fission barrier heights.

The finite-range EDF by Fayans et al. [19 21] was used first to find $Q_{\alpha}$ for new superheavy $\alpha$-chains in Ref. [22], within a partially self-consistent calculation, with an approximate consideration of the deformation energy. The total binding energy of a deformed nucleus was presented as a sum of two terms, $E_{B}(N, Z)=$ $E_{\text {sph }}(N, Z)+E_{\text {def }}(N, Z)$, where the main, spherical one, was found for a Fayans EDF, whereas the "deformation" addendum was taken from published tables for two Skyrme EDFs. To be definite, a version DF3-a [23] of the initial Fayans EDF DF3 20, 21] was used for the spherical energy. For the deformation energy, the EDF HFB-17 [24, 25] was used for nuclei with $Z \leq 110$ and MSk7 [26], for $Z>110$. Accuracy of such semi-self-consistent calculations turned out to be only a bit worse compared to that of the MMM approach.

The absence of a deformed Fayans EDF solver was the reason of the use of such non-consistent ansatz in Ref. 22. Recently, however, such a code has been developed [27]. A localized version FaNDF ${ }^{0}$ [28] of the general finite range Fayans EDF was used, making the surface term more similar to the Skyrme one. This allowed to employ, with some modifications, the computer code HFBTHO [29], developed originally for Skyrme EDFs. First applications of this code to deformed nuclei [27, 30 32. with the use of the original set of parameters of the EDF $\mathrm{FaNDF}^{0}$ [28] found for spherical nuclei turned out to be rather successful. The goal of the present work is to provide a new, completely self-consistent, calculation of $Q_{\alpha}$ energies for six superheavy $\alpha$-chains with the Fayans EDF. For a comparison, calculations with two Skyrme EDFs, SLy4 and SkM*, are carried out. These two parametrizations are rather commonly employed in various Skyrme EDF calculations. In particular, they were used as representatives of non-relativistic EDFs in [14]. Thus, this part of our calculations repeats that of [14] and partly earlier study of [33]. With these two Skyrme parametrizations, when considering low-lying collective excitations in spherical nuclei, the older $\mathrm{SkM}^{*}$ was found more successful than SLy4 [34]. Predictions of the selfconsistent methods are compared to the MMM of Refs. [10, 11].

Theoretical predictions for $Q_{\alpha}$ are important not only by itself but also to find the lifetime $T_{\alpha}$. Indeed, the latter is governed mainly by the exponential Gamow factor [35], which is found almost unambiguously in terms of $Q_{\alpha}$ by finding the penetrability of the Coulomb barrier in the daughter nucleus for an emitted alpha particle. Unfortunately, at the present there is no a reliable microscopic theory for the pre-exponential factor as this is a very complicated many-body problem. It is worth mentioning recent studies in this direction 36 39]. Some of them are rather promising but do not provide a simple tool for systematic calculations. In such a situation, the phenomenological approaches are more practical. Most of them are close to the classical Viola-Seaborg formula [40], which involves seven phenomenological parameters. Here, just as in [22], we use the five-parameter modification of this formula [41] by Parkhomenko and Sobiczewski (PS). For a comparison, similar calculations were repeated with the use of more recent 12-parameter formula by Royer and Zhang (RZ) [42].

\section{FAYANS EDF PREDICTIONS FOR $Q_{\alpha}$ VALUES IN SUPERHEAVY NUCLEI}

In this section, we give the SCMF predictions of the Fayans EDF FaNDF ${ }^{0}$ 28] for six $\alpha$-decay chains which begin from the following parent superheavy nuclei: ${ }^{294} 118,{ }^{294} 117,{ }^{293} 117,{ }^{291} \mathrm{Lv},{ }^{288} 115$, and ${ }^{287} 115$. For completeness, we write down explicitly the main ingredients of this EDF. In the Fayans method, the ground state energy of a nucleus is considered as a functional of normal density $\rho$ and anomalous density $\nu$, as

$$
E_{0}=\int \mathcal{E}[\rho(\mathbf{r}), \nu(\mathbf{r})] d^{3} r,
$$

where the isotopic indices and the spin-orbit densities are omitted for brevity.

The volume part of the EDF, $\mathcal{E}^{\mathrm{v}}(\rho)$, is taken as a fractional function of densities $\rho_{+}=\rho_{n}+\rho_{p}$ and $\rho_{-}=\rho_{n}-\rho_{p}$ :

$$
\mathcal{E}^{\mathrm{v}}(\rho)=C_{0}\left[a_{+}^{\mathrm{v}} \frac{\rho_{+}^{2}}{4} f_{+}^{\mathrm{v}}(x)+a_{-}^{\mathrm{v}} \frac{\rho_{-}^{2}}{4} f_{-}^{\mathrm{v}}(x)\right],
$$

where

$$
f_{+}^{\mathrm{v}}(x)=\frac{1-h_{1+}^{\mathrm{v}} x^{\sigma}}{1+h_{2+}^{\mathrm{v}} x^{\sigma}}
$$

and

$$
f_{-}^{\mathrm{v}}(x)=\frac{1-h_{1-}^{\mathrm{v}} x}{1+h_{2-}^{\mathrm{v}} x} .
$$

Here, $x=\rho_{+} / \rho_{0}$ is the dimensionless nuclear density, $\rho_{0}=2\left(k_{\mathrm{F}}^{0}\right)^{3} / 3 \pi^{2}$ being the equilibrium symmetric nuclear matter density. The coefficient $C_{0}=\left(d n / d \varepsilon_{\mathrm{F}}\right)^{-1}=$ $\pi^{2} /\left(k_{\mathrm{F}}^{0}\right) m$ ) is the usual normalization factor used in the theory of finite Fermi system (TFFS) [43], the inverse density of states at the Fermi surface. The power parameter $\sigma=1 / 3$ is chosen in the $\mathrm{FaNDF}^{0}$ functional, in contrast to the case for DF3 or DF3-a, where $\sigma=1$ is used. The dimensionless parameters in Eqs. 35 are the same as in [28]: $a_{+}^{\mathrm{v}}=-9.559, h_{1+}^{\mathrm{v}}=0.633, h_{2+}^{\mathrm{v}}=0.131$, $a_{-}^{\mathrm{v}}=4.428, h_{1-}^{\mathrm{v}}=0.25$, and $h_{2-}^{\mathrm{v}}=1.300$. They correspond to the following characteristics of nuclear matter: the equilibrium density $\rho_{0}=0.160 \mathrm{fm}^{-3}$ (the corresponding mean radius parameter is $r_{0}=1.143 \mathrm{fm}$ ), the energy per particle $\mu=-16.0 \mathrm{MeV}$, the incompressibility 
$K_{0}=220 \mathrm{MeV}$, and the asymmetry energy coefficient of $a_{\text {sym }}=30.0 \mathrm{MeV}$. Higher derivatives of the EDF of nuclear matter over the densities $\rho_{+}, \rho_{-}$, suggested in [17] for the Skyrme EDFs as a set of criteria for functionals, are given in [27] for the $\mathrm{FaNDF}^{0}$ functional.

If one sets $h_{2+}^{\mathrm{v}}=h_{2-}^{\mathrm{v}}=0$, the volume part of the Fayans EDF reduces to the form typical for Skyrme EDFs. As was discussed in detail in [27], the "Fayans denominator" and the use of the bare mass $m^{*}=m$, both peculiarities of the Fayans EDF method, are generically related to the self-consistent TFFS [4], reflecting in a hidden form the energy-dependence effects inherent to this approach.

Until recently, the Fayans EDF was applied to spherical nuclei only. These applications were rather successful, in comparison with analogous Skyrme Hartree-FockBogoliubov (HFB) calculations. They included the analysis of the magnetic [45, 46] and quadrupole [47, 48] moments in odd nuclei, of characteristics of the first $2^{+}$excitations in even semi-magic nuclei [49, 50], of charge radii [51], and of beta-decay [52] as well. In addition, singleparticle spectra of seven magic nuclei was described with high accuracy [53]. A short review comparison of predictions of Fayans and Skyrme EDFs for these phenomena in spherical nuclei was given in Ref. [54], and more detailed one, in Ref. [55].

The use of the local approximation for the Yukawa finite-range function, $\mathrm{Yu}(r) \rightarrow 1-r_{c}^{2} \nabla^{2}$, in the DF3-like EDFs leads to the following structure of the surface term of the $\mathrm{FaNDF}^{0}$ functional:

$$
\mathcal{E}^{\mathrm{s}}(\rho)=C_{0} \frac{1}{4} \frac{a_{+}^{\mathrm{s}} r_{0}^{2}\left(\nabla \rho_{+}\right)^{2}}{1+h_{+}^{\mathrm{s}} x^{\sigma}+h_{\nabla}^{\mathrm{s}} r_{0}^{2}\left(\nabla x_{+}\right)^{2}},
$$

with $h_{+}^{\mathrm{s}}=h_{2+}^{\mathrm{v}}, a_{+}^{\mathrm{s}}=0.600, h_{\nabla}^{\mathrm{s}}=0.440$. This approximation is the main point which permitted in [27 to modify the Skyrme HFB computer code HFBTHO 29] for the Fayans EDF. This code solves the HFB equations in axially symmetric harmonic oscillator basis by assuming time-reversal symmetry.

Here, just as in [27], we use a two-parameter form for the anomalous term of the EDF:

$$
\mathcal{E}_{\text {anom }}=C_{0} \sum_{i=n, p} \nu_{i}^{\dagger}(\mathbf{r}) f^{\xi}\left(x_{+}(\mathbf{r})\right) \nu_{i}(\mathbf{r}),
$$

where the density-dependent dimensionless effective pairing force is

$$
f^{\xi}\left(x_{+}\right)=f_{\mathrm{ex}}^{\xi}+h^{\xi} x_{+} .
$$

Two models for pairing will be used, the volume one with $h^{\xi}=0$, and the surface model, with $h^{\xi} \simeq-f_{\mathrm{ex}}^{\xi}$. As we use the zero-range pairing force, a cut-off should be used in solving the gap equation. Here we use the same cut-off energy $E_{\text {cut }}=60 \mathrm{MeV}$ as in [27]. The standard procedure to solve the HFB equations is used. The code 29] for Skyrme EDFs and its analogue for the Fayans EDF used in 27] and here makes it possible to restore particle number approximately within the Lipkin-Nogami

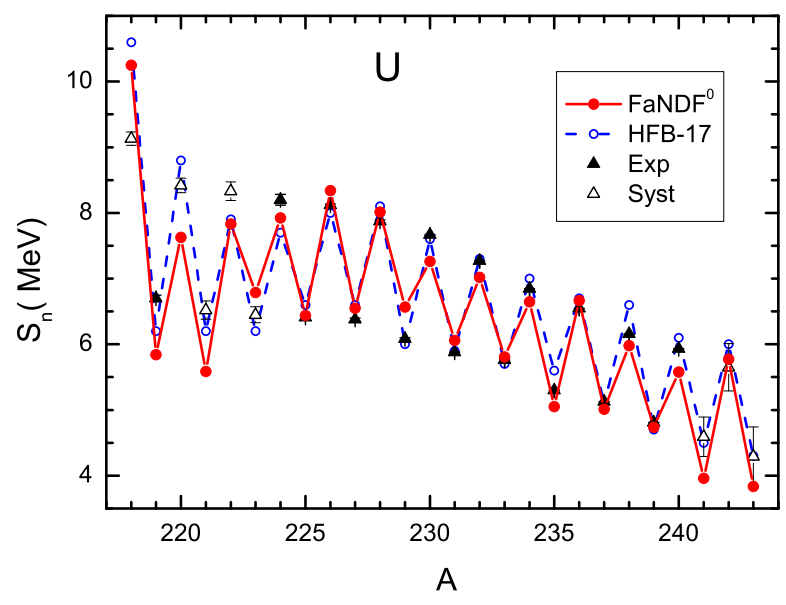

FIG. 1: The $S_{n}$ values for the U isotopic chain.

scheme. Its effect is significant for the total pairing energy but negligible (about $0.1-0.2 \mathrm{MeV}$ ) for the binding energy differences entering to the $Q_{\alpha}$ values.

For odd nuclei, we used the equal filling approximation for the quasiparticle blocking prescription when solving the HFB equations. We stress that, within this prescription, the mean-field of the odd (or odd-odd) nucleus is solved fully self-consistently. The equal filling approximation has been examined in many works, for example, in Refs. 56, 57]. In the latter, it was estimated that this approximation introduces inaccuracy of the order of $0.1-$ $0.2 \mathrm{MeV}$ for the binding energy. This is much smaller than the differences between various EDFs for the predicted $Q_{\alpha}$ values.

Pairing interaction influences significantly the oneneutron separation energies

$$
S_{n}(N, Z)=B(N, Z)-B(N-1, Z) .
$$

In figure 1 they are shown for uranium isotopes, calculated with the $\mathrm{FaNDF}^{0} \mathrm{EDF}$, with two models of pairing specified above. The corresponding values of the parameters of Eq. (8) are $\left(f^{\xi}=-0.440, h^{\xi}=0\right)$ for the volume pairing and $\left(f^{\xi}=-1.433, h^{\xi}=1.375\right)$ for the surface one. In this work, the calculation scheme for the Fayans EDF is the same as that in Ref. [27] for the uranium chain. In particular, the HFB equations were solved in a basis of 25 oscillator shells. Comparison is made with experimental data [58] and predictions from the Skyrme EDF HFB-17 EDF [24, 25]. We see that the Fayans EDF with both pairing models reproduce experimental data sufficiently well, with an accuracy comparable with that of the Skyrme EDF HFB-17.

Next, we investigate the alpha-decay energies $Q_{\alpha}$. The results of calculations are presented in figure 2 and in Table [1. For the Fayans EDF, predictions of the volume and surface pairing are given. A comparison is made with the data and predictions of two Skyrme EDFs, the SLy4 [16 and $\mathrm{SkM}^{*}$ 15. Corresponding results are found by us with the use of the code 29]. In this case, 20 oscillator 
shells were used, in accordance with [59]. For both of the Skyrme EDFs, the mixed pairing is used [29]:

$$
V_{\text {pair }}^{n, p}\left(\mathbf{r}, \mathbf{r}^{\prime}\right)=V_{0}^{n, p}\left(1-\alpha \frac{\rho(\mathbf{r})}{\rho_{c}}\right) \delta\left(\mathbf{r}-\mathbf{r}^{\prime}\right),
$$

$\alpha=0.5$, with obvious notation. The paring strength is taken to be the same for neutrons and protons, namely $V_{0}^{n}=V_{0}^{p}=-259.0 \mathrm{MeV}$. For completeness, we included also predictions of the MMM method taken from the tables in [10], $Z=102-109$, and [11], $Z=110-120$. Several empty places in the MMM column of Table $\$ are caused with absence of the corresponding values in the tables cited above.

A remark should be made about the experimental $Q_{\alpha}$ values used in the tables and figures $2-7$. They are taken mainly from the references cited in the caption of Table 1 . where the alpha-energies $E_{\alpha}$ were measured, and $Q_{\alpha}$ being found by accounting the recoil effect. This recipe may be doubtful in the case of odd and odd-odd nuclei where the $\alpha$-transition between ground states can be forbidden, and the observed transition may connect excited states. The analysis of the lifetimes $T_{\alpha}$ may help to clear up this point. They will be analyzed in the next Section. There are also cases when experimental $Q_{\alpha}$ values are not available. In this kind of situation we use estimated masses from mass systematics of Ref. [58]. They are shown with the open triangles in the figures.

By eye, the accuracy of the MMM is higher compared to self-consistent approaches. Among the latest EDFs, the Fayans one, with both the models for pairing, has the accuracy comparable with both the Skyrme ones. To estimate it quantitatively, we present in Table I the differences

$$
\delta Q_{\alpha}=Q_{\alpha}^{\text {theor }}-Q_{\alpha}^{\exp }
$$

with obvious notation. In the last column, the mark "(syst)" is used for the mass systematics data given in Ref. [58]. In the last line of the table, values are given of the root-mean-square deviation (RMSD) of the theory under consideration from the experiment:

$$
\left\langle\delta Q_{\alpha}\right\rangle_{\mathrm{rms}}=\sqrt{\frac{1}{N} \sum_{i}\left(Q_{\alpha, i}^{\mathrm{theor}}-Q_{\alpha, i}^{\exp }\right)^{2}} .
$$

From the $\delta Q_{\alpha}$ results, we can see that the MMM exceeds in accuracy all the self-consistent methods used, with its RMSD being smaller by a factor of 1.5-2. However, it is worth to note that the MMM parameters, those of the liquid-drop model and of the Saxon-Woods shellmodel potential, were fitted in Ref. [10, 11] to characteristics of heavy deformed nuclei in the uranium region, close to the nuclear map region under consideration. Per contra, the main part of the EDFs parameters is universal for all nuclei. Among the self-consistent calculations presented, the SLy4 EDF results in the highest accuracy. The Fayans EDF has approximately $10 \%$ worse RMSD.
However, it is worth reminding that the FaNDF $^{0}$ parameters were fitted in [28] to characteristics of the spherical nuclei only of the region between calcium and lead chains. Evidently, more fine tuning of this EDF parameters is necessary, including heavy deformed nuclei into the fitting procedure. Accuracy of the SkM* EDF is approximately two times worse comparing to other EDFs used.

\section{LIFETIMES $T_{\alpha}$ WITH RESPECT TO ALPHA-DECAY}

For completeness we recite the commonly used classical formula by Viola and Seaborg [40]. In particular, it was used in [3] - 6] to connect values of $Q_{\alpha}$ and $T_{\alpha}$ found experimentally. It reads:

$$
\lg T_{\alpha}(Z, N)=(a Z+b) Q_{\alpha}^{-1 / 2}+(c Z+d)+h_{i},
$$

where $a, b, c, d, h_{i}$ are adjusted parameters. Three of them, $h_{i}, i=p, n, p n$, are introduced to reproduce a change of the $\alpha$-decay lifetime in odd-proton, oddneutron and odd-odd nuclei with respect to "favored" decays of even-even nuclei with zero orbital moment $l$ of the $\alpha$-particle. The ground states of mother and daughter odd or odd-nuclei have usually different $J^{\pi}$ values, therefore the $\alpha$-transition between them will be of unfavored type with $l>0$, with additional hindrance due to penetration through the centrifugal barrier. On the other hand, a favored transition may occur to the excited state of the daughter nucleus.

An optimum set of the parameters of Eq. (13) for uranium and trans-uranium regions can be found in Ref. [41], where authors modified this formula to fiveparameter PS form:

$$
\log _{10} T_{\alpha}(Z, N)=a Z\left[Q_{\alpha}(Z, N)-\bar{E}_{i}\right]^{-1 / 2}+b Z+c,
$$

with the following set of parameters $a=1.5372, b=$ -0.1607 and $c=-36.573$. The parameter $\bar{E}_{i}$ in (14) has the meaning of the average excitation energy of the daughter nucleus, being zero in the case of even-even nuclei. For other types of nuclei, the following values of parameters were found in [41]:

$$
\begin{array}{r}
\bar{E}_{i}=\bar{E}_{p}=0.113 \mathrm{MeV} \quad \text { for odd-proton, } \\
\bar{E}_{i}=\bar{E}_{n}=0.171 \mathrm{MeV} \text { for odd-neutron, } \\
\bar{E}_{i}=\bar{E}_{p}+\bar{E}_{n} \text { for odd-odd nuclei }
\end{array}
$$

nuclei. These rather simple and transparent PS formulas were used in 22] and they are also used in the present work. The results are given in Table III. The meaning of superscripts for experimental values is the same as in Table I. For three parent nuclei, namely ${ }^{286} \mathrm{Fl},{ }^{279} \mathrm{Ds}$, and ${ }^{271} \mathrm{Sg}$, there is a strong competition between $\alpha$-decay and fission. In these cases, we gave the total lifetime values only and the $\alpha$-decay percentage only, as it was given in the original experimental works. Recently, there has 
TABLE I: $\alpha$-decay energies $Q_{\alpha}(\mathrm{MeV})$ of superheavy nuclei. In each line, $Z$ and $A$ correspond to the parent nucleus. $\operatorname{FaNDF}^{0}$ (surf) and $\mathrm{FaNDF}^{0}$ (vol) denote the Fayans EDF with the surface and volume pairing, correspondingly. Experimental data are taken from [3] $\left({ }^{a)}\right),[8]\left({ }^{b)}\right)$, 7] $\left.{ }^{(c)}\right)$, [58], compilation of several experiments, $\left({ }^{d)}\right)$, 60] $\left({ }^{e)}\right)$. The estimated data $\left({ }^{(s y s t)}\right)$ are taken from the systematics in [58].

\begin{tabular}{|c|c|c|c|c|c|c|}
\hline Nuclei & FaNDF $^{0}$ (surf) & $\operatorname{FaNDF}^{0}(\mathrm{vol})$ & SLy4 & $\mathrm{SkM}^{*}$ & MMM & Exp. \\
\hline${ }^{294} 118$ & 11.278 & 11.310 & 11.364 & 11.330 & 12.11 & $11.81(0.06)^{a)}$ \\
\hline${ }^{290} \mathrm{Lv}$ & 11.116 & 10.981 & 10.354 & 11.074 & 11.08 & $10.99(0.08)^{a)}$ \\
\hline${ }^{286} \mathrm{Fl}$ & 10.847 & 10.683 & 9.719 & 11.734 & 10.86 & $10.37(0.06)^{a)}$ \\
\hline${ }^{282} \mathrm{Cn}$ & 11.188 & 11.005 & 10.886 & 11.688 & 10.68 & $10.11(0.2)^{(\text {syst })}$ \\
\hline${ }^{278} \mathrm{Ds}$ & 11.272 & 11.026 & 10.849 & 11.802 & 10.54 & $10.37(0.2)^{\text {(syst) }}$ \\
\hline${ }^{274} \mathrm{Hs}$ & 9.497 & 9.788 & 9.870 & 9.932 & 9.55 & $9.57(0.2)^{(\mathrm{syst})}$ \\
\hline${ }^{270} \mathrm{Sg}$ & 8.913 & 9.407 & 9.004 & 9.319 & 8.74 & $8.99(0.3)^{\text {(syst) }}$ \\
\hline${ }^{266} \mathrm{Rf}$ & 7.617 & 7.316 & 7.052 & 8.508 & 7.05 & $7.55(0.3)^{\text {(syst) }}$ \\
\hline${ }^{262} \mathrm{No}$ & 7.497 & 7.210 & 6.649 & 7.932 & 6.86 & $7.25(0.3)^{\text {(syst) }}$ \\
\hline${ }^{294} 117$ & 10.225 & 10.131 & 11.629 & 11.033 & 11.43 & $11.20(0.04)^{b)}$ \\
\hline${ }^{290} 115$ & 11.117 & 10.898 & 10.143 & 10.792 & 10.65 & $10.45(0.04)^{b)}$ \\
\hline${ }^{286} 113$ & 9.494 & 9.479 & 8.792 & 10.879 & 9.98 & $9.79(0.05)^{c)}$ \\
\hline${ }^{282} \mathrm{Rg}$ & 10.524 & 10.701 & 10.037 & 11.512 & 9.85 & $9.18(0.03)^{b)}$ \\
\hline${ }^{278} \mathrm{Mt}$ & 10.334 & 9.681 & 9.851 & 10.442 & 9.55 & $9.59(0.03)^{b)}$ \\
\hline${ }^{274} \mathrm{Bh}$ & 8.957 & 9.187 & 8.911 & 9.285 & 8.83 & $8.97(0.03)^{b)}$ \\
\hline${ }^{270} \mathrm{Db}$ & 7.966 & 7.528 & 7.643 & 8.376 & 8.34 & $8.02(0.03)^{b)}$ \\
\hline${ }^{266} \mathrm{Lr}$ & 7.717 & 8.663 & 7.993 & 8.449 & 6.79 & $7.57(0.3)^{(\mathrm{syst})}$ \\
\hline${ }^{262} \mathrm{Md}$ & 6.564 & 6.431 & 6.011 & 7.621 & - & $6.5(0.3)^{(\text {syst })}$ \\
\hline${ }^{293} 117$ & 10.605 & 10.502 & 10.976 & 10.990 & 11.53 & $11.18(0.05)^{d)}$ \\
\hline${ }^{289} 115$ & 11.356 & 11.167 & 10.035 & 10.688 & 10.74 & $10.52(0.05)^{d)}$ \\
\hline${ }^{285} 113$ & 10.271 & 10.154 & 9.592 & 11.900 & 10.21 & $10.03(0.05)^{d)}$ \\
\hline${ }^{281} \mathrm{Rg}$ & 10.850 & 11.003 & 10.719 & 11.736 & 10.48 & $9.41(0.05)^{c)}$ \\
\hline${ }^{277} \mathrm{Mt}$ & 10.498 & 9.956 & 10.007 & 10.618 & 9.84 & $9.71(0.2)^{(\mathrm{syst})}$ \\
\hline${ }^{273} \mathrm{Bh}$ & 9.014 & 9.456 & 9.170 & 9.386 & 8.89 & $9.06(0.3)^{\text {(syst) }}$ \\
\hline${ }^{269} \mathrm{Db}$ & 8.596 & 8.739 & 7.902 & 8.305 & 8.17 & $8.49(0.3)^{\text {(syst) }}$ \\
\hline${ }^{265} \mathrm{Lr}$ & 7.162 & 7.021 & 7.208 & 8.503 & 6.62 & $7.23(0.2)^{\text {(syst) }}$ \\
\hline${ }^{261} \mathrm{Md}$ & 6.708 & 6.960 & 6.400 & 8.030 & - & $6.75(0.4)^{\text {(syst) }}$ \\
\hline${ }^{291} \mathrm{Lv}$ & 10.959 & 10.987 & 10.557 & 10.798 & 10.91 & $10.89(0.07)^{a)}$ \\
\hline${ }^{287} \mathrm{Fl}$ & 10.463 & 10.243 & 9.130 & 11.528 & 10.56 & $10.16(0.06)^{a)}$ \\
\hline${ }^{283} \mathrm{Cn}$ & 10.506 & 10.275 & 10.499 & 11.731 & 10.16 & $9.67(0.06)^{a)}$ \\
\hline${ }^{279} \mathrm{Ds}$ & 11.097 & 10.876 & 10.234 & 11.361 & 10.24 & $9.84(0.06)^{a)}$ \\
\hline${ }^{275} \mathrm{Hs}$ & 9.321 & 9.606 & 9.600 & 9.920 & 9.41 & $9.44(0.06)^{a)}$ \\
\hline${ }^{271} \mathrm{Sg}$ & 8.747 & 8.115 & 8.554 & 8.541 & 8.71 & $8.67(0.08)^{a)}$ \\
\hline${ }^{267} \mathrm{Rf}$ & 7.998 & 9.077 & 8.046 & 8.204 & - & $7.89(0.3)^{(\text {syst })}$ \\
\hline${ }^{263} \mathrm{No}$ & 7.031 & 6.759 & 6.287 & 8.634 & 6.45 & $7.0(0.4)^{(\mathrm{syst})}$ \\
\hline${ }^{288} 115$ & 11.376 & 10.968 & 9.389 & 11.485 & 10.95 & $10.61(0.06)^{e)}$ \\
\hline${ }^{284} 113$ & 10.661 & 10.588 & 10.393 & 11.930 & 10.68 & $10.15(0.06)^{e)}$ \\
\hline${ }^{280} \mathrm{Rg}$ & 11.208 & 11.274 & 11.023 & 11.717 & 10.77 & $9.87(0.06)^{e)}$ \\
\hline${ }^{276} \mathrm{Mt}$ & 10.681 & 10.393 & 10.490 & 10.919 & 10.09 & $9.85(0.06)^{e)}$ \\
\hline${ }^{272} \mathrm{Bh}$ & 8.978 & 9.612 & 8.836 & 9.128 & 9.08 & $9.02(0.06)^{e)}$ \\
\hline${ }^{268} \mathrm{Db}$ & 8.598 & 8.245 & 8.514 & 8.239 & 7.90 & $8.26(0.3)^{e)}$ \\
\hline${ }^{264} \mathrm{Lr}$ & 7.343 & 7.334 & 6.606 & 8.902 & 6.84 & $7.4(0.3)^{\text {(syst) }}$ \\
\hline${ }^{260} \mathrm{Md}$ & 6.823 & 7.259 & 6.919 & 8.189 & - & $6.94(0.3)^{\text {(syst) }}$ \\
\hline${ }^{287} 115$ & 11.628 & 11.362 & 9.840 & 11.772 & 11.21 & $10.59(0.09)^{e)}$ \\
\hline${ }^{283} 113$ & 11.264 & 11.090 & 10.990 & 12.269 & 11.12 & $10.12(0.09)^{e)}$ \\
\hline${ }^{279} \mathrm{Rg}$ & 10.494 & 10.607 & 11.489 & 12.107 & 11.08 & $10.37(0.16)^{e)}$ \\
\hline${ }^{275} \mathrm{Mt}$ & 11.789 & 11.726 & 10.585 & 10.909 & 10.34 & $10.33(0.09)^{e)}$ \\
\hline${ }^{271} \mathrm{Hs}$ & 9.010 & 9.760 & 9.557 & 9.425 & 9.07 & $9.49(0.16)^{(\text {syst })}$ \\
\hline${ }^{267} \mathrm{Db}$ & 8.312 & 7.667 & 7.412 & 8.378 & 7.41 & $7.9(0.3)^{\text {(syst) }}$ \\
\hline${ }^{263} \mathrm{Lr}$ & 7.742 & 7.355 & 6.979 & 8.744 & 7.26 & $7.68(0.2)^{(\mathrm{syst})}$ \\
\hline${ }^{259} \mathrm{Md}$ & 6.968 & 7.737 & 7.091 & 8.352 & - & $7.11(0.2)^{\text {(syst) }}$ \\
\hline
\end{tabular}


TABLE II: Differences in Eq. (11) between theoretical predictions of $\alpha$-decay energies of superheavy nuclei from Table 1 and the corresponding experimental data. The mark "(syst)" in the last column denotes the data from the systematics in [58].

\begin{tabular}{|c|c|c|c|c|c|c|}
\hline Nuclei & FaNDF $^{0}$ (surf) & $\mathrm{FaNDF}^{0}(\mathrm{vol})$ & SLy4 & $\mathrm{SkM}^{*}$ & MMM & data from \\
\hline${ }^{294} 118$ & -0.532 & -0.500 & -0.446 & -0.480 & 0.300 & [3] \\
\hline${ }^{290} \mathrm{Lv}$ & 0.126 & -0.009 & -0.636 & 0.084 & 0.090 & [3] \\
\hline${ }^{286} \mathrm{Fl}$ & 0.477 & 0.313 & -0.651 & 1.364 & 0.490 & [3] \\
\hline${ }^{282} \mathrm{Cn}$ & 1.078 & 0.895 & 0.776 & 1.578 & 0.570 & [58](syst) \\
\hline${ }^{278} \mathrm{Ds}$ & 0.902 & 0.655 & 0.479 & 1.432 & 0.170 & [58] (syst) \\
\hline${ }^{274} \mathrm{Hs}$ & -0.073 & 0.218 & 0.300 & 0.362 & -0.020 & [58] (syst) \\
\hline${ }^{270} \mathrm{Sg}$ & -0.078 & 0.417 & 0.014 & 0.329 & -0.250 & [58] (syst) \\
\hline${ }^{266} \mathrm{Rf}$ & 0.067 & -0.234 & -0.498 & 0.958 & -0.500 & [58] (syst) \\
\hline${ }^{262} \mathrm{No}$ & 0.247 & -0.040 & -0.601 & 0.682 & -0.390 & [58] (syst) \\
\hline${ }^{294} 117$ & -0.975 & -1.069 & 0.429 & -0.167 & 0.230 & [8] \\
\hline${ }^{290} 115$ & 0.667 & 0.448 & -0.307 & 0.342 & 0.200 & [8] \\
\hline${ }^{286} 113$ & -0.296 & -0.311 & -0.998 & 1.089 & 0.190 & [7] \\
\hline${ }^{282} \mathrm{Rg}$ & 1.344 & 1.521 & 0.857 & 2.332 & 0.670 & [8] \\
\hline${ }^{278} \mathrm{Mt}$ & 0.744 & 0.091 & 0.261 & 0.852 & -0.040 & [8] \\
\hline${ }^{274} \mathrm{Bh}$ & -0.013 & 0.217 & -0.059 & 0.315 & -0.140 & [8] \\
\hline${ }^{270} \mathrm{Db}$ & -0.054 & -0.492 & -0.377 & 0.356 & 0.320 & [8] \\
\hline${ }^{266} \mathrm{Lr}$ & 0.147 & 1.093 & 0.423 & 0.879 & -0.780 & [58] (syst) \\
\hline${ }^{262} \mathrm{Md}$ & 0.064 & -0.069 & -0.489 & 1.121 & - & [58](syst) \\
\hline${ }^{293} 117$ & -0.575 & -0.678 & -0.204 & -0.190 & 0.350 & [58] \\
\hline${ }^{289} 115$ & 0.836 & 0.647 & -0.485 & 0.168 & 0.220 & [58] \\
\hline${ }^{285} 113$ & 0.241 & 0.124 & -0.438 & 1.870 & 0.180 & [58] \\
\hline${ }^{281} \mathrm{Rg}$ & 1.440 & 1.593 & 1.309 & 2.326 & 1.070 & [7] \\
\hline${ }^{277} \mathrm{Mt}$ & 0.788 & 0.246 & 0.297 & 0.908 & 0.130 & [58] (syst) \\
\hline${ }^{273} \mathrm{Bh}$ & -0.046 & 0.396 & 0.110 & 0.326 & -0.170 & [58] (syst) \\
\hline${ }^{269} \mathrm{Db}$ & 0.106 & 0.249 & -0.588 & -0.185 & -0.320 & [58] (syst) \\
\hline${ }^{265} \mathrm{Lr}$ & -0.068 & -0.209 & -0.022 & 1.273 & -0.610 & [58] (syst) \\
\hline${ }^{261} \mathrm{Md}$ & -0.042 & 0.210 & -0.350 & 1.280 & - & [58] (syst) \\
\hline${ }^{291} \mathrm{Lv}$ & 0.069 & 0.097 & -0.333 & -0.092 & 0.020 & [3] \\
\hline${ }^{287} \mathrm{Fl}$ & 0.303 & 0.083 & -1.030 & 1.368 & 0.400 & [3] \\
\hline${ }^{283} \mathrm{Cn}$ & 0.836 & 0.605 & 0.829 & 2.061 & 0.490 & [3] \\
\hline${ }^{279} \mathrm{Ds}$ & 1.257 & 1.036 & 0.394 & 1.521 & 0.400 & [3] \\
\hline${ }^{275} \mathrm{Hs}$ & -0.119 & 0.166 & 0.160 & 0.480 & -0.030 & [3] \\
\hline${ }^{271} \mathrm{Sg}$ & 0.077 & -0.555 & -0.116 & -0.129 & 0.040 & [3] \\
\hline${ }^{267} \mathrm{Rf}$ & 0.108 & 1.187 & 0.156 & 0.314 & - & [58] (syst) \\
\hline${ }^{263} \mathrm{No}$ & 0.031 & -0.241 & -0.713 & 1.634 & -0.550 & [58] (syst) \\
\hline${ }^{288} 115$ & 0.766 & 0.358 & -1.221 & 0.875 & 0.130 & [60] \\
\hline${ }^{284} 113$ & 0.511 & 0.438 & 0.243 & 1.780 & 0.530 & [60] \\
\hline${ }^{280} \mathrm{Rg}$ & 1.338 & 1.404 & 1.153 & 1.847 & 0.900 & [60] \\
\hline${ }^{276} \mathrm{Mt}$ & 0.831 & 0.543 & 0.640 & 1.069 & 0.240 & [60] \\
\hline${ }^{272} \mathrm{Bh}$ & -0.042 & 0.592 & -0.184 & 0.108 & 0.060 & $60]$ \\
\hline${ }^{268} \mathrm{Db}$ & 0.338 & -0.015 & 0.254 & -0.021 & -0.360 & [60] \\
\hline${ }^{264} \mathrm{Lr}$ & -0.057 & -0.066 & -0.794 & 1.502 & -0.560 & [58](syst) \\
\hline${ }^{260} \mathrm{Md}$ & -0.117 & 0.319 & -0.021 & 1.249 & - & [58] (syst) \\
\hline${ }^{287} 115$ & 1.038 & 0.772 & -0.750 & 1.182 & 0.620 & [60] \\
\hline${ }^{283} 113$ & 1.144 & 0.970 & 0.870 & 2.149 & 1.000 & [60] \\
\hline${ }^{279} \mathrm{Rg}$ & 0.124 & 0.237 & 1.119 & 1.737 & 0.710 & [60] \\
\hline${ }^{275} \mathrm{Mt}$ & 1.459 & 1.396 & 0.255 & 0.579 & 0.010 & [60] \\
\hline${ }^{271} \mathrm{Bh}$ & -0.480 & 0.270 & 0.067 & -0.065 & -0.420 & [58](syst) \\
\hline${ }^{267} \mathrm{Db}$ & 0.412 & -0.233 & -0.488 & 0.478 & -0.490 & [58](syst) \\
\hline${ }^{263} \mathrm{Lr}$ & 0.062 & -0.325 & -0.701 & 1.064 & -0.420 & [58](syst) \\
\hline${ }^{259} \mathrm{Md}$ & -0.142 & 0.627 & -0.019 & 1.242 & - & [58] (syst) \\
\hline$\left\langle\delta Q_{\alpha}\right\rangle_{\mathrm{rms}}=$ & 0.643 & 0.647 & 0.593 & 1.148 & 0.450 & \\
\hline
\end{tabular}




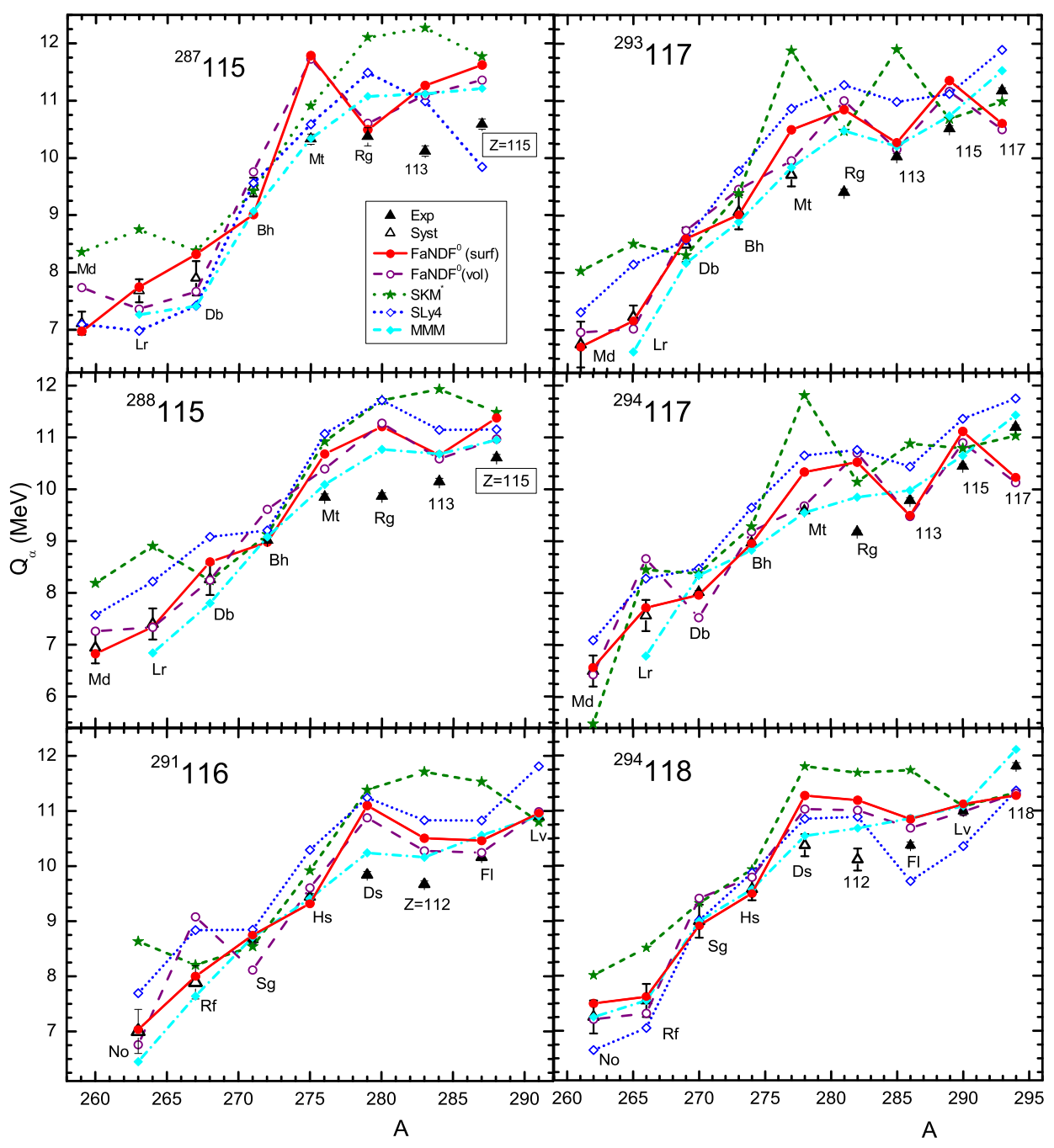

FIG. 2: The $Q_{\alpha}$ values for alpha decay chains starting from nuclei ${ }^{287} 115,{ }^{293} 117,{ }^{288} 115,{ }^{294} 117,{ }^{291} \mathrm{Lv}$, and ${ }^{294} 118$. Theoretical results are shown for FaNDF ${ }^{0}$ with surface and volume pairing, for SkM* and SLy4 Skyrme EDFs, and for the MMM model. These are compared to experimental and estimated values.

been several theoretical analysis of such competition, see e.g. 62] and references therein.

Since the formula (15) for $T_{\alpha}$ is essentially empirical, it is reasonable to examine some alternative for it. The empirical formula for $T_{\alpha}$ of favored $\alpha$-transitions by Royer [61], or its modification by Royer and Zhang [42], has the form of

$$
\log _{10} T_{\alpha}(Z, N)=a+b A^{1 / 6}+\frac{c Z}{\sqrt{Q_{\alpha}}}
$$

Here the coefficients $a, b, c$ are different for four different kinds of nuclei, see Table IV] The abbreviation 'eo' means even $Z$, odd $N$, and so on.

The unfavored $\alpha$-transitions occur in odd and oddodd nuclei, provided that the $J^{\pi}$ values of the parent and daughter nuclei do not coincide, resulting in the $\alpha$ particle orbital moment $l$ being nonzero. Several generalizations of Eq. (16), which take into account additional hindrance due to the contribution of the centrifugal bar- rier, have been presented 63 65, 67]. Here, we use a simple parameter free ansatz for this $l$-dependent addendum by Dong et al. [67]:

$$
\log _{10} T_{\alpha}(l)=\log _{10} T_{\alpha}(l=0)+\frac{l(l+1)}{\sqrt{(A-4)(Z-2) A^{-2 / 3}}} .
$$

For the first term of this formula the RZ recipe, Eq. (16), was used.

Recently Wang et al. 68] used a modification of Eq. (17), with four additional parameters for the $l$-dependent term. In this work, the role of the centrifugal term was examined in detail. In the sample of $341 \alpha$-transitions, with $J^{\pi}$ values known from [63] for both the parent and daughter nuclei, the average difference

$$
\langle\delta \lg T\rangle_{\mathrm{rms}}=\sqrt{\sum_{i}\left(\log _{10} T_{\alpha}^{\text {theor }}-\log _{10} T_{\alpha}^{\exp }\right)^{2} / N}
$$


TABLE III: The $\log _{10} T_{\alpha}$ for superheavy nuclei found with the PS formula [41], Eq. (14). The meaning of the upper labels for experimental data is the same as in Table [ The asterisks indicate three cases, where the decay is mixed and the total lifetime is given. The percent number shows the weight of the $\alpha$-transition.

\begin{tabular}{|c|c|c|c|c|c|c|c|}
\hline Nuclei & FaNDF $^{0}$ (surf) & $\mathrm{FaNDF}^{0}(\mathrm{vol})$ & SLy4 & $\mathrm{SkM}^{*}$ & MMM & $\log _{10} T_{\alpha}\left(Q_{\alpha}^{\exp }\right)$ & $\exp$ \\
\hline${ }^{294} 118$ & -1.52 & -1.60 & -1.73 & -1.65 & -3.41 & $-2.75(0.13)$ & $-3.23-(-2.71)^{a)}$ \\
\hline${ }^{290} \mathrm{Lv}$ & -1.73 & -140 & 0.20 & -1.63 & -1.64 & $-1.43(0.23)$ & $-2.27-(-1.99)^{a)}$ \\
\hline${ }^{286} \mathrm{Fl}$ & -1.68 & -128 & 1.32 & -3.73 & -1.72 & $-0.47(0.16)$ & $-0.96-(-0.77)^{a *},[50 \%]$ \\
\hline 294117 & 1.67 & 1.94 & -1.98 & -0.52 & -1.50 & $-0.94(0.10)$ & $-1.51-(-0.84)^{b)}$ \\
\hline${ }^{290} 115$ & -1.34 & -0.79 & 1.25 & -0.52 & -0.15 & $0.39(0.11)$ & $-0.10-0.56^{b)}$ \\
\hline \multirow[t]{2}{*}{${ }^{286} 113$} & 2.51 & 0.26 & 4.82 & -1.37 & 1.05 & $1.61(0.15)$ & $0.62-0.91^{b)}$ \\
\hline & & & & & & & $0.60-1.11^{c)}$ \\
\hline${ }^{282} \mathrm{Rg}$ & -1.09 & -1.54 & 0.23 & -3.49 & 0.76 & $2.80(0.10)$ & $2.06-2.72^{b)}$ \\
\hline${ }^{278} \mathrm{Mt}$ & -1.24 & 0.57 & 0.08 & -1.52 & 0.96 & $0.84(0.09)$ & $0.34-1.00^{b)}$ \\
\hline${ }^{274} \mathrm{Bh}$ & 2.08 & 1.36 & 2.23 & 1.06 & 2.50 & $2.04(0.10)$ & $1.26-1.92^{b)}$ \\
\hline${ }^{270} \mathrm{Db}$ & 4.79 & 6.52 & 6.05 & 3.29 & - & $4.58(0.12)$ & $3.33-4.02^{b)}$ \\
\hline${ }^{293} 117$ & 0.15 & 0.42 & -0.81 & -0.84 & -2.15 & $-1.31(0.12)$ & $-1.41-(-1.02)^{d)}$ \\
\hline${ }^{289} 115$ & -2.33 & -1.88 & 1.07 & -0.69 & -0.83 & $-0.26(0.13)$ & $-0.31-0.18^{d)}$ \\
\hline${ }^{285} 113$ & -0.23 & 0.09 & 1.69 & -4.14 & -0.07 & $0.43(0.14)$ & $0.69-1.08^{c)}$ \\
\hline${ }^{291} \mathrm{Lv}$ & -0.924 & -0.995 & 0.12 & -0.52 & -0.801 & $-0.75(0.18)$ & $-1.92-(-1.40)^{a)}$ \\
\hline${ }^{287} \mathrm{Fl}$ & -0.269 & 0.325 & 3.65 & -2.89 & -0.524 & $0.55(0.17)$ & $-0.43-(-0.19)^{a)}$ \\
\hline${ }^{283} \mathrm{Cn}$ & -1.02 & -0.409 & -1.00 & -3.93 & -0.098 & $1.29(0.18)$ & $0.49-0.70^{a)}$ \\
\hline${ }^{279} \mathrm{Ds}$ & -3.09 & -2.57 & -0.95 & -3.70 & -0.962 & $0.13(0.18)$ & $-0.80-(-0.60)^{a *)},[10 \%]$ \\
\hline${ }^{275} \mathrm{Hs}$ & 0.955 & 0.120 & 0.14 & -0.76 & 0.690 & $0.60(0.18)$ & $-0.92-(-0.39)^{a)}$ \\
\hline${ }^{271} \mathrm{Sg}$ & 2.03 & 4.20 & 2.67 & 2.71 & 2.15 & $2.29(0.17)$ & $1.89-2.41^{a *)},[70 \%]$ \\
\hline${ }^{288} 115$ & -1.97 & -0.97 & $3.53-2.23$ & -0.93 & $-0.04(0.16)$ & $-1.24-(-0.72)^{e)}$ & \\
\hline${ }^{284} 113$ & -0.81 & -0.62 & $-0.10-3.83$ & -0.86 & $0.57(0.17)$ & $-0.51-0.03^{e)}$ & \\
\hline${ }^{280} \mathrm{Rg}$ & -2.79 & -2.94 & $-2.34-3.95$ & -1.72 & $0.70(0.17)$ & $0.36-0.90^{e)}$ & \\
\hline${ }^{276} \mathrm{Mt}$ & -2.13 & -1.39 & $-1.64-2.71$ & -0.58 & $0.08(0.17)$ & $-0.33-0.20^{e)}$ & \\
\hline${ }^{272} \mathrm{Bh}$ & 2.02 & 0.09 & 2.481 .54 & 1.69 & $1.88(0.19)$ & $0.80-1.33^{e)}$ & \\
\hline$\langle\delta \lg T\rangle_{\mathrm{rms}}=$ & 1.52 & 1.53 & 1.89 & 2.46 & 0.70 & 0.33 & \\
\hline
\end{tabular}

between the theoretical predictions (with the experimental values of $Q_{\alpha}$ used) and the experimental values for the lifetimes were found in different models. At first, the initial Royer formula (16) was used and then, the one (17) with the $l$-dependent term included. It turned out that the gain for the due to the $\langle\delta \lg T\rangle_{\mathrm{rms}}$ value is about 0.1 . To be more exact, from $\langle\delta \lg T\rangle_{\mathrm{rms}}=0.587$ without the $l$-term to $\langle\delta \lg T\rangle_{\mathrm{rms}}=0.481$ with it. Of course, this gain is different for different kinds of nuclei. It is equal to zero for even-even nuclei, being about 0.2 for odd-odd ones. The use of the modified centrifugal term in 68] results in a rather small additional gain, $\langle\delta \lg T\rangle_{\mathrm{rms}}=0.433$. The corresponding values of $\langle\delta \lg T\rangle_{\mathrm{rms}}=0.536$ in 64 and $\langle\delta \lg T\rangle_{\mathrm{rms}}=0.561$ in [65] are also given in [68]. The

TABLE IV: The coefficients [42] of the RZ formula (16).

\begin{tabular}{|c|c|c|}
\hline $\mathrm{a}$ & $\mathrm{b}$ & $\mathrm{c}$ \\
\hline ee -25.31 & -1.1629 & 1.5864 \\
\hline eо -26.65 & -1.0859 & 1.5848 \\
\hline oe -25.68 & -1.1423 & 1.592 \\
\hline оo -29.48 & -1.113 & 1.6971 \\
\hline
\end{tabular}

predictions for lifetimes found in this work with the use of different theoretical values of $Q_{\alpha}$ are essentially worse. For example, for the Skyrme HFB-27 EDF the average deviation from the experiment $\langle\delta \lg T\rangle_{\mathrm{rms}}=1.617$ was found. The values of $\langle\delta \lg T\rangle_{\mathrm{rms}}$ found with Eq. (18) for each theoretical column in Table III are given in the last line of this table. In this calculation, we excluded three cases of mixed decays.

In contrast to [68], the present work addresses odd and odd-odd superheavy nuclei for which the characteristics are often not known from the experiment. Therefore, it is reasonable to use for systematic calculations of the $\log _{10} T_{\alpha}\left(Q_{\alpha}^{\exp }\right)$ values Eq. (17) assuming that all the $\alpha$ transitions are favored, i.e. putting $l=0$. Thus, we use, in fact, the RZ formula (16). In Table V] we present the results of such calculations for the same set of nuclei as in Table III. In both of the tables, we found from Eq. (18) for each kind of the theory used the average difference between the theoretical prediction for $\log _{10} T_{\alpha}$ and the corresponding experimental value, three cases of mixed decays again being excluded from the averaging procedure. A comparison of the values of $\langle\delta \lg T\rangle_{\mathrm{rms}}$ in the columns of $\log _{10} T_{\alpha}\left(Q_{\alpha}^{\exp }\right)$ in tables III and $\mathrm{V}$ is a 
TABLE V: The $\log _{10} T_{\alpha}$ values for superheavy nuclei found with the RZ formula [42], Eq. (16). The meaning of the upper labels for the experimental data is the same as in tables 1 and 3.

\begin{tabular}{|c|c|c|c|c|c|c|c|}
\hline Nuclei & $\mathrm{FaNDF}^{0}$ (surf) & $\mathrm{FaNDF}^{0}(\mathrm{vol})$ & SLy4 & $\mathrm{SkM}^{*}$ & MMM & $\log _{10} T_{\alpha}\left(Q_{\alpha}^{\exp }\right)$ & $\exp$ \\
\hline${ }^{294} 118$ & -2.14 & -2.22 & -2.35 & -2.27 & -4.09 & $-3.41(0.14)$ & $-3.23-(-2.71)^{a)}$ \\
\hline${ }^{290} \mathrm{Lv}$ & -2.34 & -2.00 & -0.34 & -2.23 & -2.25 & $-2.02(0.20)$ & $-2.27-(-1.99)^{a)}$ \\
\hline${ }^{286} \mathrm{Fl}$ & -2.27 & -1.85 & 0.83 & -4.39 & -2.30 & $-1.02(0.16)$ & $\left.-0.96-(-0.77)^{a *}\right),[50 \%]$ \\
\hline${ }^{294} 117$ & 1.57 & 1.86 & -2.30 & -0.75 & -1.79 & $-1.19(0.11)$ & $-1.51-(-0.84)^{b)}$ \\
\hline${ }^{290} 115$ & -1.65 & -1.07 & 1.09 & -0.78 & -0.38 & $0.19(0.11)$ & $-0.10-0.56^{b)}$ \\
\hline${ }^{286} 113$ & 2.39 & 2.44 & 4.83 & -1.71 & 0.86 & $1.44(0.16)$ & $0.62-0.91^{b)}$ \\
\hline & & & & & & & $0.60-1.11^{c)}$ \\
\hline${ }^{282} \mathrm{Rg}$ & -1.44 & -1.92 & -0.05 & -3.99 & 0.51 & $2.67(0.11)$ & $2.06-2.72^{b)}$ \\
\hline${ }^{278} \mathrm{Mt}$ & -1.62 & 0.29 & -0.23 & -1.92 & 0.69 & $0.57(0.09)$ & $0.34-1.00^{b)}$ \\
\hline${ }^{274} \mathrm{Bh}$ & 1.85 & 1.09 & 2.01 & 0.77 & 2.29 & $1.81(0.10)$ & $1.26-1.92^{b)}$ \\
\hline${ }^{270} \mathrm{Db}$ & 4.66 & 6.47 & 5.98 & 3.10 & 3.23 & $4.45(0.12)$ & $3.33-4.02^{b)}$ \\
\hline${ }^{293} 117$ & -0.33 & -0.05 & -1.30 & -1.34 & -2.67 & $-1.82(0.13)$ & $-1.41-(-1.02)^{d)}$ \\
\hline${ }^{289} 115$ & -2.85 & -2.39 & 0.62 & -1.18 & -1.31 & $-0.73(0.13)$ & $-0.31-0.18^{d)}$ \\
\hline${ }^{285} 113$ & -0.70 & -0.38 & 1.26 & -4.68 & -0.53 & $-0.03(0.14)$ & $0.69-1.08^{c)}$ \\
\hline${ }^{291} \mathrm{Lv}$ & -1.22 & -1.30 & -0.18 & -0.81 & -1.10 & $-1.05(0.18)$ & $\left.-1.92-(-1.40)^{a}\right)$ \\
\hline${ }^{287} \mathrm{Fl}$ & -0.57 & 0.02 & 3.36 & -3.22 & -0.83 & $0.25(0.17)$ & $-0.43-(-0.19)^{a)}$ \\
\hline${ }^{283} \mathrm{Cn}$ & -1.33 & -0.72 & -1.32 & -4.27 & -0.41 & $0.98(0.18)$ & $0.49-0.70^{a)}$ \\
\hline${ }^{279} \mathrm{Ds}$ & -3.43 & -2.90 & -1.27 & -4.04 & -1.29 & $-0.19(0.17)$ & $-0.80-(-0.60)^{a *)},[10 \%]$ \\
\hline${ }^{275} \mathrm{Hs}$ & 0.63 & -0.20 & -0.19 & -1.08 & 0.37 & $0.28(0.18)$ & $\left.-0.92-(-0.39)^{a}\right)$ \\
\hline${ }^{271} \mathrm{Sg}$ & 1.71 & 3.88 & 2.35 & 2.39 & 1.83 & $1.96(0.27)$ & $1.89-2.41^{a *)},[70 \%]$ \\
\hline${ }^{288} 115$ & -2.29 & -1.22 & 3.54 & -2.56 & -1.17 & $-0.60(0.17)$ & $-1.24-(-0.72)^{e)}$ \\
\hline${ }^{284} 113$ & -1.08 & -0.88 & -0.33 & -4.29 & -1.13 & $0.38(0.18)$ & $-0.51-0.03^{e)}$ \\
\hline${ }^{280} \mathrm{Rg}$ & -3.20 & -3.37 & -2.73 & -4.44 & -2.07 & $0.49(0.18)$ & $0.36-0.90^{e)}$ \\
\hline${ }^{276} \mathrm{Mt}$ & -2.53 & -1.75 & -2.02 & -3.15 & -0.89 & $-0.19(0.18)$ & $-0.33-0.20^{e)}$ \\
\hline${ }^{272} \mathrm{Bh}$ & 1.82 & -0.21 & 2.30 & 1.32 & 1.48 & $1.68(0.20)$ & $0.80-1.33^{e)}$ \\
\hline$\langle\delta \lg T\rangle_{\mathrm{rms}}=$ & 1.67 & 1.64 & 1.87 & 2.82 & 0.87 & 0.23 & \\
\hline
\end{tabular}

direct comparison of the accuracy of Eq. (15) from [41] and Eq. (16) from [42]. We see that the latter is a bit more accurate that is not strange as it contains twelve fitted parameters in comparison with five ones in the first case.

We can now compare the accuracy of the different theoretical methods in the description of the $\alpha$-decay lifetimes. We see that both the methods to calculate the lifetimes lead to approximately the same accuracy, the Parkhomenko-Sobiczewski method turning out to be a bit better. Evidently, the accuracy of the RZ method could be made higher if all the $\alpha$-transitions were not considered as favored. Again, the MMM approach is more accurate than all the self-consistent methods used. For this characteristic, the Fayans method with the FaNDF ${ }^{0}$ EDF and both models for pairing exceed a bit in accuracy the SLy4 EDF and significantly the SkM* one. Note that the $\langle\delta \lg T\rangle_{\mathrm{rms}}$ value for the Fayans EDF is approximately the same as that found in 68] for the HFB-27 EDF, the most accurate member of the family in predicting nuclear masses Skyrme EDFs.

In order to estimate the role of the $l$-dependent term in Eq. (17) we chose three $\alpha$-decays in the chain of ${ }^{293} 117$ for which we obtained $\log _{10} T_{\alpha}\left(Q_{\alpha}^{\exp }\right)<\log _{10} T_{\alpha}^{\exp }$. Due to hindrance effect of the centrifugal barrier, its inclusion may improve the agreement with the data. The calculated results are given in TableVI We see that, indeed, the inclusion of the $l$-dependent term does improve the situation with the use of Eq. (17). The values of $\log _{10} T_{\alpha}\left(Q_{\alpha}^{\exp }\right)$ agree with the data at $l=3, l=4$ for the ${ }^{293} 117$ and ${ }^{289} 115$ parent nuclei and at $l=4, l=5$ for the ${ }^{285} 113$ one. Unfortunately, this remedy can help only in the cases where the inequality $\log _{10} T_{\alpha}\left(Q_{\alpha}^{\exp }\right)<$ $\log _{10} T_{\alpha}^{\exp }$ holds, whereas the opposite sign of this inequality takes place in many cases in Table $\mathrm{V}$. For example, this happens for the ${ }^{275} 108$ nucleus for which we obtained $\log _{10} T_{\alpha}\left(Q_{\alpha}^{\exp }\right)-\log _{10} T_{\alpha}^{\exp }=0.487$. However, as the analysis in [68] showed the average accuracy of Eq. (17) and analogous formulas in 68] for $\log _{10} T_{\alpha}$ are of the order of 0.5 , the disagreement under discussion is not extraordinary.

\section{CONCLUSION}

Alpha-decay energies $Q_{\alpha}$ for several chains of superheavy nuclei are found within the SCMF approach by employing the Fayans functional $\mathrm{FaNDF}^{0}$. Two models for 
TABLE VI: The role of the $l$-dependent term in formula (17). The meaning of the upper labels for the experimental data is the same as in Table

\begin{tabular}{cccc}
\hline \hline Nuclei & 1 & $\log _{10} T_{\alpha}\left(Q_{\alpha}^{\exp }\right)$ & $\exp$ \\
\hline${ }^{293} 117$ & 0 & -1.82 & $-1.41-(-1.02)^{d)}$ \\
& 1 & -1.74 & \\
& 2 & -1.60 & \\
& 3 & -1.38 & \\
& 4 & -1.09 & \\
& 5 & -0.72 & \\
\hline${ }^{289} 115$ & 0 & -0.73 & $-0.31-0.18^{d)}$ \\
& 1 & -0.66 & \\
& 2 & -0.51 & \\
& 3 & -0.29 & \\
& 4 & 0.01 & \\
& 5 & 0.37 & \\
\hline 285113 & 0 & -0.03 & $0.69-1.08^{c)}$ \\
& 1 & 0.05 & \\
& 2 & 0.20 & \\
& 3 & 0.42 & \\
& 4 & 0.72 & \\
& 5 & 1.09 & \\
\hline \hline
\end{tabular}

the effective pairing force were used, the volume and the surface pairing. The results are compared to the experimental data and predictions of two Skyrme functionals, SLy4 16] and SkM* 15]. Predictions of the macro-micro method [10, 11] are also considered. The Fayans EDF results in the average deviation from experimental energies by $\left\langle\delta Q_{\alpha}\right\rangle_{\mathrm{rms}}^{\text {tot }}=0.643 \mathrm{MeV}$ with the surface pairing and $0.647 \mathrm{MeV}$ with the volume pairing. These values are slightly larger than the corresponding SLy4 value of 0.593 $\mathrm{MeV}$ but significantly less than the $\mathrm{SkM}^{*}$ value of 1.148 $\mathrm{MeV}$. However, in this problem all the considered selfconsistent methods give in the MMM, the corresponding value being only $0.454 \mathrm{MeV}$. It is worth stressing that the $\mathrm{FaNDF}^{0}$ EDF used here was found in [28] by adjusting to masses and radii of spherical nuclei only, from the calcium to the lead region. Therefore a readjustment of its parameters with the use of all the nuclear chart is desirable. As a first step in this direction, we plan to adjust spin-orbit and effective tensor constants similarly to that made in 23] for spherical nuclei, which turned out to be successful. As a second step, the optimization of the EDF parameters at deformed HFB level, by utilizing input data on heavy nuclei, similarly to [66], should be performed.

The corresponding $\alpha$-decay lifetimes are calculated with the use of the semi-phenomenological five-parameter PS formula [41], or, alternatively, the twelve-parameter RZ one [42]. The role of the $l$-dependent term for the unfavored $\alpha$-transitions in the form of [67] was also examined. The accuracy of the method itself to calculate the lifetimes can be estimated by comparing the $\log _{10} T_{\alpha}\left(Q_{\alpha}^{\exp }\right)$ values with the experimental data of $\log _{10} T_{\alpha}$. For the bulk of $24 \alpha$-transitions between superheavy nuclei we examined, the RZ formula gives an av- erage deviation of $\langle\delta \lg T\rangle_{\mathrm{rms}}=0.23$, whereas it is equal to 0.33 for the PS case. Thus, the first method looks more accurate itself. However, the accuracy of all calculations with the use of theoretical $Q_{\alpha}$ values turns out a bit worse in the RZ case.

Any inaccuracy in finding $Q_{\alpha}$ leads to a defect in reproducing the $T_{\alpha}$ values, the scale of disagreement being even enhanced. Again, the MMM exceeds in accuracy all the self-consistent calculations. The corresponding value of $\langle\delta \lg T\rangle_{\mathrm{rms}}$ is equal to 0.69 with the use of the PS formula and to 0.87 , with the RZ one. In this problem the Fayans approach has some advantage over the other self-consistent methods used. For this, we obtained $\langle\delta \lg T\rangle_{\mathrm{rms}} \simeq 1.50$ with the PS formula and $\simeq 1.65$ for the RZ one. For comparison, the corresponding values are 1.89 and 1.87 for the SLy4 EDF, and also 2.46 and 2.82 for the $\mathrm{SkM}^{*}$ one. Note that the average deviation from experiment for the Skyrme HFB-27 EDF found in [68] is $\langle\delta \lg T\rangle_{\mathrm{rms}} \simeq 1.5$, i.e. it is comparable to that for the Fayans EDF.

In conclusion, we should again stress that readjustment of the Fayans EDF parameters with the use of wider bulk of nuclei, including the deformed and heavy ones, is necessary to attempt to reach the level of accuracy in reproducing the $Q_{\alpha}$ and $T_{\alpha}$ values comparable to that of the MMM.

\section{ACKNOWLEDGMENT}

This work was supported with Grants Nos. 16-1210155 and 16-12-10161 from Russian Science Foundation. It was also partly supported by the RFBR Grant 16-02-00228. This work was also supported (M.K.) by the Academy of Finland under the Centre of Excellence Programme 2012-2017 (Nuclear and Accelerator Based Physics Programme at JYFL) and FIDIPRO programme. Calculations are partially made on the Computer Center of NRC "KI". 
[1] W. D. Myers and W. J. Swiatecki, Nucl. Phys. 81, 1 (1966).

[2] V.M. Strutinskii, Sov. J. Nucl. Phys. 3, 449 (1966).

[3] Yu. Ts. Oganessian et al., Phys. Rev. C 74, 044602 (2006).

[4] Yu. Ts. Oganessian et al., Phys. Rev. Lett. 104, 142502 (2010).

[5] Yu. Ts. Oganessian et al., Phys. Rev. C 83, 054315 (2011).

[6] Yu. Ts. Oganessian et al., Phys. Rev. Lett. 108, 022502 (2012).

[7] Yu. Ts. Oganessian et al., Phys. Rev. C 87, 054621 (2013).

[8] J. Khuyagbaatar et al., Phys. Rev. Lett. 112, 172501 (2014).

[9] Yu. Ts. Oganessian et al., Phys. Rev. C 79, 024603 (2009).

[10] I. Muntian, S. Hofmann, Z. Patyk, and A. Sobiczewski, Acta Phys. Polon. B 34, 2073 (2003).

[11] I. Muntian, Z. Patyk, and A. Sobiczewski, Phys. At. Nucl. 66, 1015 (2003).

[12] P. Möller, J. R. Nix, W. D. Myers, and W. J. Swiatecki, At. Data Nucl. Data Tables 59, 185 (1995).

[13] P. Möller, et.al, At. Data Nucl. Data Tables 109-110, 1 (2016).

[14] P.-H. Heenen, J. Skalski, A. Staszczak, D. Vretenar, Nucl. Phys. A 944, 415 (2015).

[15] J. Bartel, P. Quentin, M. Brack, C. Guet, and H. B. Håkansson, Nucl. Phys. A 38679 (1982).

[16] E. Chabanat, P. Bonche, P. Haensel, J. Meyer, and R. Schaeffer, Nucl. Phys. A 635, 231 (1998).

[17] M. Dutra, O. Lourenceo, J. S. Sá Martins, A. Delfino, J. R. Stone, and P. D. Stevenson, Phys. Rev. C 85, 035201 (2012).

[18] T. Niks̃ić, D. Vretenar, P. Ring, Phys. Rev. C 78 (2008) 034318.

[19] A. V. Smirnov, S. V. Tolokonnikov, and S. A. Fayans, Sov. J. Nucl. Phys. 48, 995 (1988).

[20] I. N. Borzov, S. A. Fayans, E. Kromer, D. Zawischa, Z. Phys. A 355, 117 (1996).

[21] S. A. Fayans, S. V. Tolokonnikov, E. L. Trykov, and D. Zawischa, Nucl. Phys. A 676, 49 (2000).

[22] S. V. Tolokonnikov, Yu. S. Lutostansky, E. E. Saperstein, Phys. Atom. Nucl. 78, 708 (2013).

[23] S. V. Tolokonnikov and E. E. Saperstein, Phys. At. Nucl. 73, 1684 (2010).

[24] S. Goriely, N. Chamel, and J. M. Pearson, Phys. Rev. Lett. 102, 152503 (2009).

[25] Goriely S http://www-astro.ulb.ac.be/bruslib

[26] S. Goriely, F. Tondeur, and J. M. Pearson, At. Data Nucl. Data Tables 77, 311 (2001).

[27] S. V. Tolokonnikov, I. N. Borzov, M. Kortelainen, Yu. S. Lutostansky, and E. E. Saperstein, J. Phys. G, 42, $075102(2015)$.

[28] S. A. Fayans, JETP Letters 68, 169 (1998).

[29] M. V. Stoitsov, N. Schunck, M. Kortelainen, N. Michel, H. Nam, E. Olsen, J. Sarich, and S. Wild, Comp. Phys. Comm. 184, 1592 (2013).

[30] S. V. Tolokonnikov, I. N. Borzov, M. Kortelainen, Yu. S. Lutostansky, and E. E. Saperstein, EPJ Web of Conferences, 107, 02003 (2016).
[31] E. E. Saperstein, I. N. Borzov, Yu. S. Lutostansky, and S. V. Tolokonnikov, JETP Lett. 102, 421 (2015).

[32] S. V. Tolokonnikov, I. N. Borzov, M. Kortelainen, Yu. S. Lutostansky, and E. E. Saperstein, Phys. At. Nucl. 79, No. 1, pp. 2137 (2016).

[33] S. Ćwiok, W. Nazarewicz, and P. H. Heenen, Phys. Rev. Lett. 83, 1108 (1999).

[34] J. Terasaki, J. Engel, and G. F. Bertsch, Phys. Rev. C 78, 044311 (2008).

[35] G. Gamow, Z. Phys. 51, 204 (1928).

[36] S. Peltonen, D. S. Delion, and J. Suhonen, Phys. Rev. C 78, 034608 (2008).

[37] R. I. Betan and W. Nazarewicz, Phys. Rev. C 86, 034338 (2012).

[38] D. S. Delion and A. Dumitrescu, Phys. Rev. C 87, 044314 (2013).

[39] D. E. Ward, B. G. Carlsson, and S. Aberg, Phys. Rev. C 88, 064316 (2013).

[40] V. E. Viola, Jr. and G. T. Seaborg, J. Inorg. Nucl. Chem. 28, 741 (1966).

[41] A. Parkhomenko and A. Sobiczewski, Acta Phys. Pol. B 36, 3095 (2005).

[42] G. Royer, H. F. Zhang, Phys. Rev. C 77, 037602 (2008).

[43] A. B. Migdal, Theory of finite Fermi systems and applications to atomic nuclei (Nauka, Moscow, 1965; Wiley, New York, 1967).

[44] V. A. Khodel and E. E. Saperstein, Phys. Rep. 92, 183 (1982).

[45] I. N. Borzov, E. E. Saperstein, and S. V. Tolokonnikov, Phys. At. Nucl. 71, 469 (2008).

[46] I. N. Borzov, E. E. Saperstein, S. V. Tolokonnikov, G. Neyens, and N. Severijns, Eur. Phys. J. A 45, 159 (2010).

[47] S. V. Tolokonnikov, S. Kamerdzhiev, S. Krewald, E. E. Saperstein, and D. Voitenkov, EPJA 48, 70 (2012).

[48] S. Kamerdzhiev, S. Krewald, S. Tolokonnikov, E. E. Saperstein, and D.Voitenkov. EPJ Web of Conferences 38, 10002 (2012).

[49] S. V. Tolokonnikov, S. Kamerdzhiev, D. Voytenkov, S. Krewald, and E. E. Saperstein, Phys. Rev. C 84, 064324 (2011).

[50] S. V. Tolokonnikov, S. Kamerdzhiev, S. Krewald, E. E. Saperstein and D. Voitenkov. EPJ Web of Conferences 38, 04002 (2012).

[51] E. E. Saperstein, S. V. Tolokonnikov, Phys. At. Nucl. 74, 1277 (2011).

[52] I. N. Borzov, Phys. Rev. C 67, 025802 (2003); Phys. Rev. C 71, 065801 (2005).

[53] N. V. Gnezdilov, I. N. Borzov, E. E. Saperstein, and S. V. Tolokonnikov, Phys. Rev. C 89, 034304 (2014).

[54] E. E. Saperstein and S. V. Tolokonnikov, EPJ Web of Conferences, 107, 02001 (2016).

[55] E. E. Saperstein and S. V. Tolokonnikov, Phys. At. Nucl. 79, 1030 (2016).

[56] S. Perez-Martin and L. M. Robledo, Phys. Rev. C 78, 014304 (2008).

[57] N. Schunck, J. Dobaczewski, J. McDonnell, J. Mor?e, W. Nazarewicz, J. Sarich, and M. V. Stoitsov, Phys. Rev. C 81, 024316 (2010).

[58] M. Wang, G. Audi, A. H. Wapstra, F. G. Kondev, M. MacCormick, X. Xu, and B. Pfeiffer, Chinese Physics C, 36, 1603 (2012). 
[59] http://massexplorer.frib.msu.edu/content/DFTMassTables.ht6̆n]] G. Royer, Nucl. Phys. A 848, 279 (2010).

[60] Yu. Ts. Oganessian et al., Phys. Rev. C 72, $034611 \quad$ 66] M. Kortelainen, T. Lesinski, J. Moré, W. Nazarewicz, J. (2005).

[61] G. Royer, J. Phys. G: Nucl. Part. Phys. 26, 1149 (2000).

[62] Y. Qian et al., Phys. Rev. C 90, 064308 (2014).

[63] V. Yu. Denisov and A. A. Khudenko, At. Data Nucl. Data Tables 95, 815 (2009).

[64] V. Yu. Denisov and A. A. Khudenko, Phys. Rev. C 79, 054614 (2009). Sarich, N. Schunck, M. V. Stoitsov, and S. Wild, Phys. Rev. C 82, 024313 (2010).

[67] J. M. Dong, et al., Nucl. Phys. A 832, 198 (2010).

[68] Z. Y. Wang, Z. M. Niu, Q. Liu, and J. Y. Guo, J. Phys. G 42, 055112 (2015). 